\title{
Hematological Changes in Dogs and Cats With Ectoparasitosis in Northern Trans-Urals
}

\author{
Yulya Tkacheva \\ Department of Anatomy and Physiology \\ FSBEI HE Northern Trans-Ural SAU, \\ Tyumen, Respubliky7, Russia, \\ E-mail: tkacheva.ya@asp.gausz.ru
}

\author{
Larisa Glazunova \\ Associate Professor \\ ASRIVEA - Branch of Tyumen Scientific Centre SB RAS \\ 2 Institutskay, Tyumen, Russia, \\ FSBEI HE Northern Trans-Ural SAU, \\ Tyumen, Respubliky7, Russia, \\ E-mail:glazunovala@gausz.ru
}

\begin{abstract}
Skin is the most vulnerable and extensive body area in mammals. The clinical manifestation of dermatopathies may vary from minor irritation to extensive ulcers causing severe discomfort and disturbance of homeostasis. The aim of the research was to study the hematological status of dogs and cats with the most common ectoparasitosis of varying severity.

The research was conducted in the period from 2015 to 2017 on the basis of the Department of Anatomy and Physiology of Northern Trans-Ural State Agricultural University and several veterinarian clinics of the city of Tyumen and the Tyumen region. For the indicated period, 19,226 domestic carnivores were studied out of which 8,602 were cats and 10,624 were dogs.

It has been stated that in the case of diseases caused by ectoparasites (ticks: Sarcoptes, Notoedres, Demodex, Ixodes and Dermacentor, as well as fleas (Ctenocephalides canis) and C. felis) serious hematologic changes occur. Blood cells actively react to the painful processes occurring in the domestic carnivores; therefore, there is a quantitative change that gives important clinical and diagnostic value for determining the severity of disease and assigning of adequate treatment.

The phenomena of erythrocytopenia occurred in dogs and cats under demodicosis, as well as during the ixodid tick attack. Erythrocytosis was observedduring notoedrosis in cats. The number of erythrocytes at that time has increased by $20 \%$. In case of ectoparasitic lesions, the level of hemoglobin tended to decrease. So, ixodidoses, flea invasion and demodicosis in dogs and cats were accompanied by anemic symptoms, which in our opinion were caused by blood loss and destruction of red blood cells by biological toxins. Leukocytosis was observed in domestic carnivores affected by pruritic ticks (notoedrosis, sarcoptosis). In some cases, the amount of leukocytes increased 2.3 times. The rate of erythrocyte sedimentation at most arachnoentomoses (flea invasion of dogs and cats, dog sarcoptosis and ixodidosis) had a stable tendency to increase, which indicates the development of necrotic region, inflammatory reaction and destructive processes in sick animals.
\end{abstract}

Key words-dogs; cats; dermatitis; hematological parameters; ectoparasites

\section{INTRODUCTION}

Ectoparasites mostly affect the skin and the hair; in some cases they may cause aneczema, itch and hair loss [3]. A large number of veterinary drugs with the insecticidal and acaricidal actions are used in veterinary practice. The need for these drugs is due to the fact that the diseases caused by the insects and ticks are invasive and cause significant damage to animal health [1].

It should be noted that ectoparasitosis not only worsens the appearance and changes the behavior of animals, but there alsooccur morphofunctional changes in animals, which often manifest as exhaustion, anemia and reduced resistance of the body [12].

The most common animal ectoparasitosis in the Northern Trans-Ural Region is sarcoptic mange, ear mange, demodectic mange, flea dermatitis and ixodidoses.

Sarcoptic (itch) mange is one of the main causes of intense itching. The disease may become chronic [10]. The causative agent of highly contagious dermatosis (which is mostly observed among dogs) is the Sarcoptes scabiei tick. This causative agent was described in the first half of the XIX ${ }^{\text {th }}$ century in Paris by the founder of the first dermatological school Jean-Louis-Marc-Aliber [6]. An infection occurs when a sick animal gets in contact with a healthy one, as well as it gets in contact with contaminated objects (trays, carriers, etc.) [8]. Demodectic mange is a parasitic dermatosis which occurs as a result of hair follicleinvasion by microscopic vermiform tick(Demodex canis) [3]. This causative agent is a symbiont and is present in the normal microflora of animal skin. The clinical signs occur when the resistance of animal's organism is decreased. Demodectics can be both local and generalized, it may have a juvenile nature (from 3 to 10 months old) or it may first occur in sexually mature animals, and the clinical picture may significantly change.The difference between these forms is relatively provisory, local demodicosis is defined as a condition under which there are ' 6 or less lesions smaller than $2.5 \mathrm{~cm}$ in diameter'. Generalized demodicosis can be considered as a condition under which there are more than 12 affected areas or lesions of the entire anatomical region (for example, head and muzzle) [7]. It is worth noting that some dogs are predisposed to a hereditary defect of T-cell immunity of varying degrees of complexity. It is not recommended to use such animals for breeding $[13,15]$. The causative agent lives in the sebaceous glands and hair follicles of skin, where it feeds on epithelial cells. The development of this disease is associated with immunodeficiency [8].There is a placental way of transferring ticks from the female to the newborn animals due to the increased activation of ticks under the effect of the hormone oxytocin which is released during the childbirth. The 
first skin lesions by this kind of ticksusually appear on the head, near the eyes, lips, on the paws, between fingers and elbows $[13,15]$. Significant factors in pathogenesis include violation of skin barrier functions, inflammation caused by bacterial infection, and type IV hypersensitivity reactions that can explain alopecia, itching, erythema, and comedones [2]. In the last few years, the newly discovered ticks and new forms of previously discovered ticks have been described [15, 17]. A distinctive feature of the tick of the genus Demodex compared to the sarcoptic mange would be the absence of itching (the itching does not always occur during this disease).

Fleas are the groups of bloodsucking insects. The causative agents in dogs are Ctenocephalides canis and in cats they would be $C$. felis. In case when domestic carnivores suffer from fleas, the disease is called ctenocephalides. The flea invasion is of great importance in veterinary medicine, since it leads to pathogenic activity on host's organism and is a carrier of various diseases. The adult fleas are obligate insects, which parasitize on warm-blooded animals. Female fleas are quite prolific and make egg laying in the external environment and the skin of the host, which soon dissipate in the environment. Having overcome the development stages from the egg to the adult, the imago uses its oral apparatus to bite the skin of the animal. It injects saliva into the wound, which contains components that prevent blood clotting and manifest allergic activity.Depending on the temperature and humidity of the environment, the development cycle of fleas from the egg to the adult takes 12-30 days [3]. The bites of fleas and the influence of more than 15 aggressive chemical components of their saliva causes sensibilization of animal's body [5]. Soreness and fever are typical clinical signs in animals infected with fleas [4]. By parasitizing on animals, fleas inflict painful bites, resulting in papules, developing dermatitis (second infection) [10]; besides, fleas may also cause itching, inflammation and peeling [9]. This disease is characterized by the loss of hair, wounds from scratchesin the upper body and lower back. Secondary infection is caused by an excessive reproduction of bacteria of Staphylococcus fungi of the genus Malassezia (commensal skin microorganisms, auditory canals, muzzle, nose, perianal surfaces of healthy dogs and cats) [12]. This disease is characterized by clinical signs in combination with animal fleas or their fecal on the animal body.

Ixodes ticks parasitize on the body of animals walking around in the city parks, squares, in the forest and fields. Ixodes ticks often wait for their prey at the top of the grass and attach themselves to the animal when it directly contactthe plant. At the time of the skin puncture the tick injects a substance that anesthesizes the attachment site and reduces the blood coagulability [12]; thus, the animal does not experience any discomfort and does not feel the tick's presence. The wound, which appears as a result of the bite of a tickmay become inflamed and cause soreness. Quite often the lipomas are formed in the spot of a tick's bite $[13,15]$.In the region of Northern Trans-Urals the Ixodes are the carriers and reservoirs of babesiosis pathogens (Babesia spp.), anaplasmosis, as well as tularemia, borreliosis and tick-borne encephalitis. In addition, a massive attack of ixodes provokes the development of ixodidosis and may even lead to the death of an animal. There are two periods in the Northern Trans-Urals when ticks become very active, i.e. spring period and summer and fall period [19, 20,21].

These ectoparasites are animal blood-feeders, secreting saliva in the dermis, which contains biologically active substances that cause irritation or provoke an allergic reaction. Besides the discomfort, serious hematologic changes are often observed in animals, which provoke the development of a secondary microflora and in some cases leads to death as a result of septicemia. The most common laboratory method indicating changes in the body of an animal is the blood study. Hematologic studies can reveal the severity of a specific disease and correct the therapy in time, taking into account the state of the animal.

Being the most important components of the internal environment of the body, blood cellsreact sensitively to various physiological and pathological processes in the body, which makes it possible to use them as a diagnostic tool in a wide variety of nosological forms [18]. The mechanism of hematological disorders atdermatopathy of parasitic etiology in dogs and cats has not been adequately studied and the relevance of this issue will affect the targeted pathogenetic therapy of domestic carnivores.

Objective: to study the hematological status of dogs and cats in the most common ectoparasitosis of different severity.

\section{MATERIALS AND METHODS}

The studies were conducted in the period of 2015-2017 on the basis of the Department of Anatomy and Physiology of Northern Trans-Ural State Agricultural University and several veterinarian clinics of the city of Tyumen and the Tyumen region.

For the indicated period, 19,226 domestic carnivores were studied out of which 8,602 were cats and 10,624 were dogs. The pet owners complained about cutaneous pathology and illbeing. All animals underwent common clinical studies, which included the collection of anamnesis, examination, as well as microscopic examination of skin using the method of deep and superficial scrapings. Deep skin scrapings were taken from the area of 3-4 $\mathrm{cm}^{2}$ from 5 parts of animal's body until the appearance of capillary blood. The surface scrapings of the skin were taken from a larger area in the direction of the hair growth; then the material was placed on a glass slide covered with a cover glass and was examined with a slight magnification of 5 or 10 times.

All clinically ill animals with final diagnosis of flea dermatitis, sarcoptic mange, notoedrosis, otodectosis, demodicosis and ixodidosis had gone through the hematological studies. A general blood test is a screening that detects many abnormalities and pathological conditions [12]. The material of the study was venous blood. The blood was taken from the subcutaneous vein of the forearm (v. cephalicaantebrachii). The biomaterial (blood) was placed in special test tubes and immediately sent to the laboratory.During and after blood collection antiseptics were applied. To assess the morphological composition of the blood of domestic carnivores, a number of indicators were studied among which were the following: the number of erythrocytes, 
leukocytes and hemoglobin which were counted with the help of an automatic analyzer BC-2800Vet, Mindray, ESR was studied with the help of an accelerated method in capillaries inclined at $60^{\circ}$ (Panchenkov method). The results were processed statistically by means of computer, the Biostat program and MicrosoftExcel.

\section{RESULTS OF RESEARCH}

During the study 8,602 cats were examined. Out of this number 764 cats had skin diseases $(8.88 \%)$, among which 68 $(8.90 \%)$ cats had final diagnosis of notoedric mange, 27 $(3.53 \%)$ cats had the diagnosis of demodicosis, and 680 $(7.91 \%)$ cats were diagnosed with otodectosis. For the current period a total number of 10,624 dogs was examined, including $1,347(12.6 \%)$ dogs with the skin diseases. 47 (3.48\%) dogs were diagnosed with sarcoptic mange; the demodicosis was registered in 34 cases $(2.52 \%)$, otodectosis ears were diagnosed in $19(0.17 \%)$ dogs out of the number of the examined dogs. The ixodid ticks were found in 1,036 animals, which was $9.75 \%$ of all dogs that were examined.

All animals that have gone through the examination and who had some skin diseases had typical clinical signs, such as hyperemia of skin areas, the formation of nodules, crusts, alopecia, etc. Thereby, the specific clinical signs of the diseases, in some cases, were not observed. There were animals who had not received any treatment for a long period of time, thereby, contributing to the development of secondary microflora with the formation of local purulent dermatitis, and, in some cases, generalized pyoderma. Therefore, the collection of anamnestic data, as well as simple physical examination of an animal is not enough to diagnose parasitic dermatitis. All animals were free from helminth infections and were vaccinated against infectious diseases. The temperature was takenand the parameters had extreme fluctuations of $37.5-39.5^{\circ} \mathrm{C}$ (within physiological norm). Visible mucous membranes had a pale pink stain. All animals with the determined diagnosis received an appropriate treatment.

In the course of hematological studies it was established that ectoparasitoses in domestic carnivores had a significant effect on certain blood parameters. Clinical studies of the blood of dogs with flea invasion indicated that parasitizing fleas did not significantly affect the animal's body. All the dog's blood values were within the limits of physiological norm and during the study period they changed insignificantly. It should be noted that in dogs with a high degree of invasion, the ESR was slightly increased to $3.6 \pm 0.4 \mathrm{~mm} / \mathrm{h}$ compared to the control group of clinically healthy dogs (TABLE II.).It was also noted that in cats with a weak flea invasion no significant changes in hematological parameters were recorded (TABLE I). With a significant degree of invasion in cats, the hemoglobin level was reduced to $90.6 .1 \mathrm{~g} / \mathrm{l}$. It can be concluded that parasitizing on cats and $\operatorname{dogs} C$. canis and C. felis have a pathogenic effect on the body leading to the shifts in some indicators due to inflammatory and allergic reactions.

TABLE I. DYNAMICS OF HEMATOLOGICAL INDICES IN CATS WITH ECTOPARASITOSES

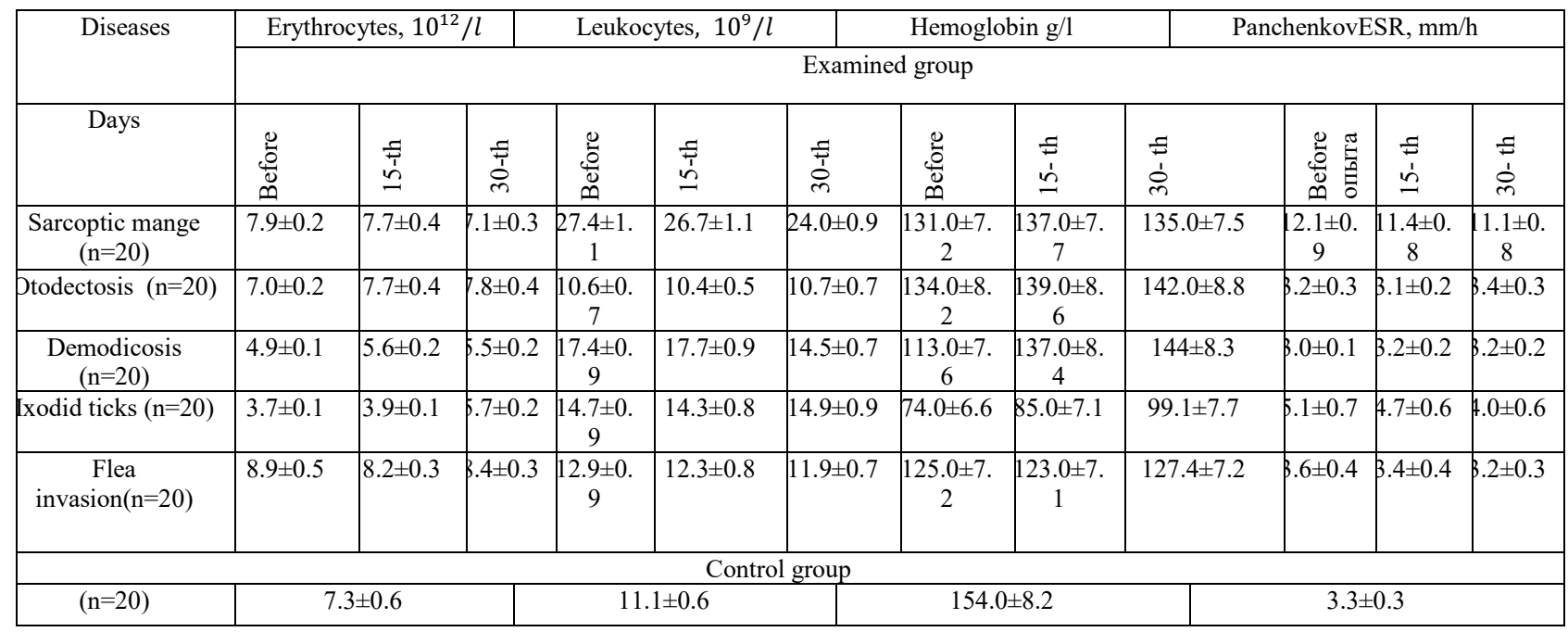

Analyzing the hematological studies of cats afflicted with notoedric mange caused by Notoedres only a slight increase in leukocytes was observed. Cats with the generalized form of notoedrosis had significant changes in blood, which were characterized by leukocytosis $18.4 \pm 0.9 \times 10^{9} / 1$, as well as an increase in the number of erythrocytes (erythrocytosis) by $20 \%$ $\left(12,6 \pm 0,8 \times 10^{12} / 1\right)$ with insignificant increase in hemoglobin up to $158 \pm 8.4 \mathrm{~g} / 1$. 
TABLE II. DYNAMICS OF HEMATOLOGICAL INDICES IN DOGS WITH ECTOPARASITOSES

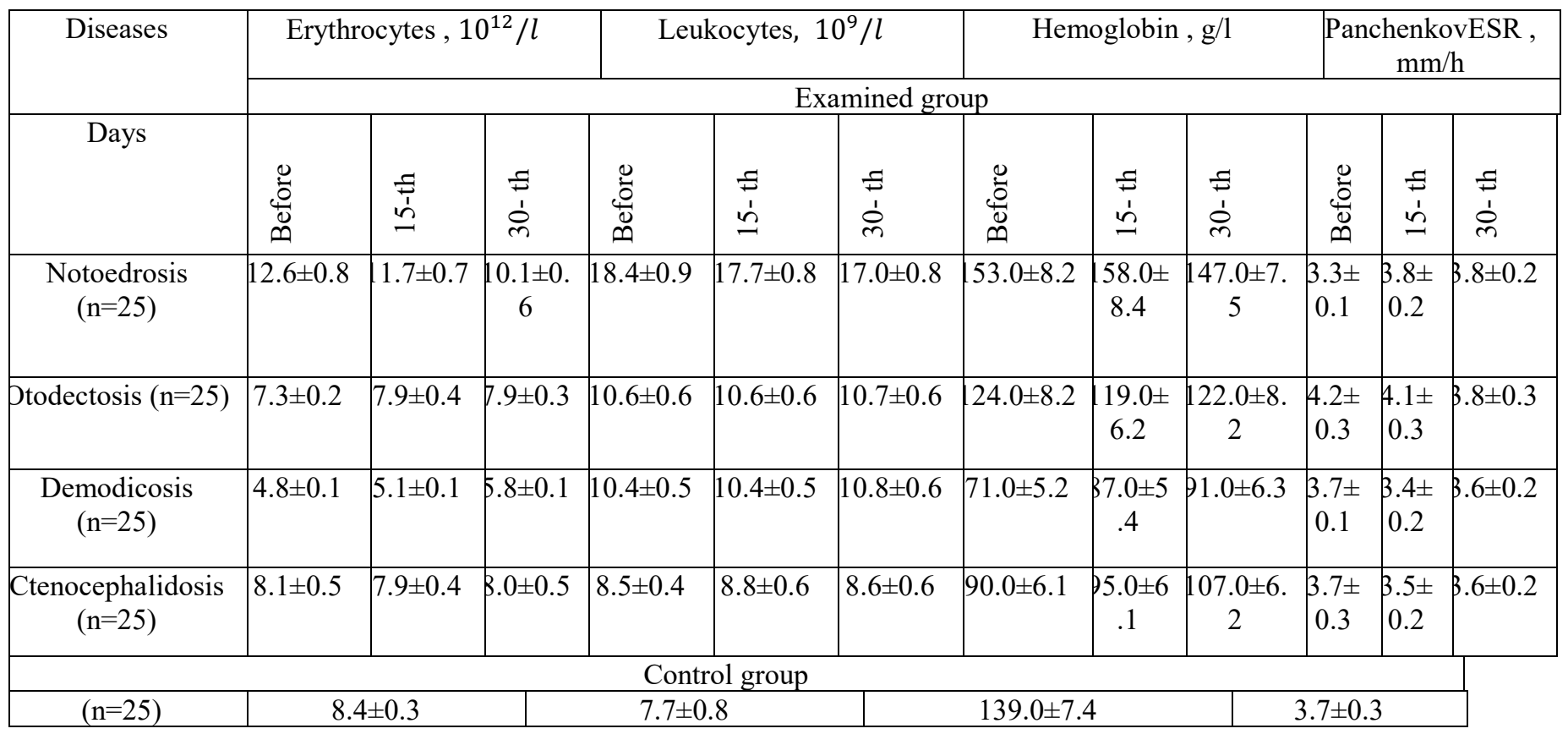

Dogs with sarcoptoses had leukocytosis $\left(27,4 \pm 1,1 * 10^{9} /\right.$ l), the number of leukocytes increased 2.3 times. Although not being a specific indicator, the ESR can indicate the inflammatory processes in dogs affected by pruritic tick. This indicator increased 3.6 times $(12.1 \pm 0.9 \mathrm{~mm} / \mathrm{h})$ compared to the control group of animals. Other indicators were in the range of physiological norm.

The results of study of cats afflicted with ticks(Demodex) showed low hemoglobin count in the blood of sick cats which was about $81 \pm 5.2 \mathrm{~g} / \mathrm{l}$ (standard rate is $80-150 \mathrm{~g} / \mathrm{l}$ ); the erythrocytes up to $4.8 \pm 0.1 * 10^{12} / l$ (standard rate is $3-10 * 10^{12} /$ l), which is below the control group of cats. Before the beginning of a treatment process, the demodecosis in dogs was characterized by a decrease in the number of red blood cells downto $4.9 \pm 0,1 * 10^{12} / l$. The leukocytosis $\left(17.7 \pm 0.9 * 10^{9} / l\right)$ was also determined, which indicates the inflammatory processes. The number of leukocytes increased 1.5 times. There was anemia in dogs before receiving therapeutic care; the hemoglobin was reduced to $(113 \pm 7.6 \mathrm{~g} / 1)$.

When dogs were afflicted with ticks(Otodectes), no obvious hematological changes were observed; all the indices were in the physiological norm. As for the cats afflicted with ticks(Otodectes), the ESR has increased slightly up to $4.2 \pm 0.3$ $\mathrm{mm} / \mathrm{h}$ compared to the control group of cats.

The affection of dogs with ixodid ticks is characterized by serious hematological disorders, namely, a decrease in the number of erythrocytes down to $3.7 \pm 0.1 * 10^{12} / l$, rapid decreases in hemoglobin to the lower limit of physiological norm $(74.7 \pm 6.6 \mathrm{~g} / \mathrm{l})$. The ESR has increased 1.6 times $(5.1 \pm 0.7$ $\mathrm{mm} / \mathrm{h}$ ). The number of leukocytes has increased slightly staying within the physiological norm $\left(14.9 \pm 0.9 \times 10^{9} / 1\right)$.

\section{CONCLUSION}

In the course of the studies it was revealed that serious hematologic changes were caused by ectoparasites (ticks: Sarcoptes, Notoedres, Demodex, Ixodes and Dermacentor, as well as Ctenocephalides canisfelis and $C$. fleas). The blood cells actively reacted to the painful processes occurring in domestic carnivores, so there was a quantitative change, and they gave an important clinical and diagnostic value for determining the severity of disease.

The phenomena of erythrocytopenia were observed in dogs and cats under demodicosis, as well as during the attack of ixodid ticks. The erythrocytosis was recorded in cats with notoedric mange with the increased number of erythrocytes up to $20 \%$, which indicated endo- or exogenous intoxications stimulating hemopoiesis. In case of ectoparasitic lesions, the hemoglobin tended to decrease. So, ixodidoses, flea invasion and demodicosis in dogs and cats were accompanied by anemic features, which in our opinion were caused by blood loss and destruction of red blood cells by biological toxins.Leukocytosis was observed in domestic carnivores affected by acarids (notoedric mange, sarcoptosis). In some casesthere was an increase of leukocytes (2.3 times). The rate of erythrocyte sedimentation at most arachnoentomoses (flea invasion of dogs and cats, sarcoptic dogs and ixodidosis) had a stable tendency to increase, which indicated the development of necrotic regions in sick animals, inflammatory reaction and destructive processes.

\section{References}

[1] M.V. Arisov, G.B. Arisova, A.V. Loganov, «Thestudyofacuteandresidualacaricidalactionof insakar preparation in laboratory conditions») Theory and practice of parasitic animal diseases, vol. 12, pp. 24-26, 2011. 
[2] L Ferrer, I Ravera, K Silbermayr, «Immunology and pathogenesis of canine demodicosis», Vet Dermatol, 2014;25:427, pp.65

[3] D.Grange, F. Heimann, Encyclopedia of the dog. Part 1,2010, pp. 402.

[4] M. R. Lappin, «Update on flea and tick associated diseases of cats», Veterinary Parasitology, vol. 254, 30 April 2018, pp. 26-29

[5] N.A. Lavrova, «Complex approach to protection of dogs and cats from parasitic diseases» Russian Veterinary Journal Small domestic and wild animals, 2006, vol. 1, pp.143-145.

[6] Yu. Maklin, Veterinary Focus, Vol. 25 (2), 2015, pp.1. Ewan McNeill BVMS, Cert VR, MRCVS - Editor in Chief

[7] W. Miller, C. Griffin, K.Campbell, «Parasitic Skin Diseases», In: Muller and Kirk`s Small Animals Dermatology. Vol.7, Toronto: Elsevier Inc, 2013, pp.304-315.

[8] L.S. Moiseenko, «Skin diseases of dogs and cats: treatment and prevention», 2016, p.187.

[9] T. Nuttall, «Skin diseases of dogs», Moscow, Aquarium LTD, 2007, p.25

[10] S. Paterson, «Skin diseases of dog». Moscow, Aquarium LTD, 2000, pp.5-8.

[11] P. Prelo, «Application of serological tests for skin diseases of dogs and cats», Veterinary focus vol. 18, pp. 24-31.

[12] J .Schissler, «Manual on the diagnosis of skin diseases in dogs», $2017 \mathrm{p}$. 25 .

[13] L. Skipin, E. Gaevaya, E. Zaharova, V. Petukhova, K. Sidorova "Biogeochemistry of heavy metals in trophic chain in terms of the south of Tumen region", Procedia Engineering (see in books). Vol. 165. pp. $860-868,2016$
[14] O.A. Stolbova, Yu.V. Glazunov, L.N. Skosyrskikh, "Ticks-parasites of dogs in Northern Trans-Urals", Indo American journal of pharmaceutical sciences. vol. 05 (03), pp.1675-1682, 2018.

[15] M. Yu. Tohou, S. N. Lutsuk, Yu.V. Dyachenko, Phenology of ticks of the genus Dermacentor in the Central Ciscaucasia, Parasitology,2013, Vol. 47, № 6, pp. 437-447.

[16] M.N. Sotskaya, «Skin and coat of a dog. Scientific, veterinary and cosmetology aspects», Moscow, Aquarium LTD, 2006, p. 225.

[17] M.Uillard, «Laboratory diagnostics in the clinic of small pets» Moscow, Aquarium LTD, 2004, p. 26.

[18] S. Waisglass, «How I approach... Demodicosis» Veterinary Focus, Vol. 25 (2), 2015, p.1.Ewan McNeill BVMS, Cert VR, MRCVS - Editor in Chief.

[19] Yu.A Watnikov, «Comparative analysis of structural changes in erythrocytes in laboratory animals under the influence of trauma» Russian Veterinary Journal (Small Pets), 2012 vol.3, pp. 20-22.

[20] L.A. Glazunova, Yu.V Glazunov, A.A. Ergashev, "Ecological-epizootical situation on telasiosis among large cattle in Northern Ural region", Research Journal of Pharmaceutical, Biological and Chemical Sciences. T. 9. Vol. 4. pp. 1687-1693, 2018.

[21] Yu. V. Glazunov, L. A. Glazunova, Changes of sexstructure of population of ixodid ticks under the action of abiotic factors, Journal of Pharmaceutical Sciences and Reserch, Vol. 10(1), 2018, pp. 129-131 